\title{
Empoderamiento en estudiantes universitarios de enfermería desde la percepción del clima organizacional
}

Empowerment in university nursing students from the perception of the organizational climate

Empoderamento em estudantes de enfermagem universitária a partir da percepção do clima organizacional

\author{
Rossana Mirtha Scarsi Maratuech \\ roscarsi76@yahoo.es \\ https://orcid.org/0000-0002-4306-7382
}

Universidad Nacional del Centro del Perú, Perú

\section{Guillermina Delia Pérez Gutarra}

delia1999@hotmail.com

https://orcid.org/0000-0002-6091-4344

Universidad Nacional de Moquegua, Ilo - Perú

\author{
Carolina Mercedes Cristobal Tembladera \\ ccristobalt@gmail.com \\ https://orcid.org/0000-0001-6690-7033 \\ Universidad Nacional de Moquegua, Ilo - Perú
}

Recibido 4 de octubre 2021 / Arbitrado y aceptado 12 de noviembre 2021 / Publicado 30 de diciembre 2021

\begin{abstract}
RESUMEN
El empoderamiento en estudiantes universitarios de enfermería, es una oportunidad para posicionar la profesión. Objetivo. Determinar la relación que existe entre empoderamiento y clima organizacional en estudiantes universitarios de enfermería. Materiales y Métodos. Estudio correlacional, transversal, realizado en Huancayo, Perú, entre marzo y diciembre de 2018 . La población estuvo conformada por 189 estudiantes de enfermería, y el tamaño de la muestra $(n=166)$ se obtuvo con la fórmula para muestras finitas y el muestreo fue aleatorio simple. Se aplicó un cuestionario validado por cada variable. Para el análisis de datos, se empleó la estadística descriptiva e inferencial, se trabajó con el software IBM SPSS Statistics 24, se aplicó para la contratación de hipótesis el coeficiente de correlación de Pearson (r). Resultados. El 58,43\% y el 39,15\%, muestran un nivel de empoderamiento medio y alto y el $84,34 \%$ perciben que el clima organizacional en el espacio académico es medianamente favorable, lo que determina una relación directa entre el empoderamiento y clima organizacional en estudiantes de enfermería (0,335), significativa $(p=0,000<0,05)$. Conclusiones. Se demuestra que existe relación directa entre empoderamiento y clima organizacional, al evidenciar un alto porcentaje de estudiantes universitarios de enfermería que perciben nivel medio en ambas variables, lo cual puede ser considerado como una brecha para el desarrollo personal y profesional del estudiante y de la organización.
\end{abstract}

Palabras clave: Estudiantes de enfermería; Empoderamiento; Organizacional; enfermería

\begin{abstract}
Empowerment in university nursing students is an undeniable opportunity to position the profession. Objective. To determine the relationship between empowerment and organizational climate in university nursing students. Materials and methods. Crosssectional correlational study carried out in Huancayo, Peru, between March and December 2018. The population consisted of 189 nursing students, and the sample was 166, simple random probability sampling. A validated questionnaire was applied for each variable. Descriptive and inferential statistics were used for data analysis, the IBM SPSS Statistics 24 software was used, and Pearson's correlation coefficient ( $r$ ) was applied for contracting hypotheses. Results. $58,43 \%$ and $39,15 \%$ show a medium and high level of empowerment and $84,33 \%$ perceive that the organizational climate in the academic space is moderately favorable, which determines a direct relationship between empowerment and organizational climate in nursing students $(0,335)$, significant $(p=$ $0,000<0,05)$. Conclusions. It is shown that there is a direct relationship between empowerment and organizational climate, by showing a high percentage of university nursing students who perceive a medium level in both variables, which can be considered as a gap for the development personal and professional of the student and the organization.
\end{abstract}

Key words: nursing students, empowerment, organizational, nursing 


\section{RESUMO}

O empoderamento dos estudantes universitários de enfermagem é uma oportunidade para posicionar a profissão. Objetivo. Determinar a relação entre o empoderamento e o clima organizacional dos estudantes universitários de enfermagem. Materiais e métodos. Estudo transversal e correlacional realizado em Huancayo, Peru, entre março e dezembro de 2018. A população consistia de 189 estudantes de enfermagem, e o tamanho da amostra $(n=166)$ foi obtido com a fórmula de amostra finita e a amostragem foi simples e aleatória. Um questionário validado foi aplicado para cada variável. Para análise de dados, foram usadas estatísticas descritivas e inferenciais, trabalhando com o software IBM SPSS Statistics 24, e o coeficiente de correlação ( $r$ ) de Pearson foi aplicado para o teste de hipóteses. Resultados. 58,43\% e $39,15 \%$ mostram um nível médio e alto de empoderamento e $84,34 \%$ percebem que o clima organizacional no espaço acadêmico é moderadamente favorável, o que determina uma relação direta entre empoderamento e clima organizacional nos estudantes de enfermagem $(0,335)$, significativo $(p=0,000<$ $0,05)$. Conclusões. Demonstra-se que existe uma relação direta entre empoderamento e clima organizacional, mostrando uma alta porcentagem de estudantes de enfermagem universitários que percebem um nível médio em ambas as variáveis, o que pode ser considerado como uma lacuna para o desenvolvimento pessoal e profissional do estudante e da organização.

Palavras-chave: Estudantes de enfermagem; Capacitação; Organizacional; Enfermagem; Capacitação

\section{INTRODUCCIÓN}

La profesión de enfermería es una de las carreras más demandadas a nivel mundial, debido entre otros factores, a la necesidad de los sistemas de salud de contar con profesionales capaces de gestionar el cuidado humano con alta calidad. En Perú, existe 143 universidades, de las cuales 24 públicas y 42 privadas ofertan la carrera profesional de enfermería, con 37, 405 estudiantes matriculados, se considera a enfermería como la segunda carrera de ciencias de la salud más demandada en el país (1).

En el contexto, las instituciones de educación superior universitaria tienen como propósito la formación de profesionales altamente competitivos, capaces de coadyuvar al desarrollo de la sociedad, por lo que es necesario realizar procesos de evaluación continua de la calidad educativa, considera al estudiante como el principal factor estratégico de este proceso (2) en el que, la valoración del clima organizacional en la gestión universitaria se convierte en una necesidad y es considerado como una herramienta de diagnóstico meritoria para impulsar procesos de cambio y elemento fundamental que trasciende al desempeño profesional (3) y puede facilitar el empoderamiento estudiantil.

Desde esta perspectiva, una de las preocupaciones fundamentales de las instituciones de educación superior en Perú, como en otros países, es mejorar la calidad de la formación de profesionales en enfermería, por lo que se enfrenta nuevos retos, como el de empoderar a los estudiantes, para que a futuro asuman diferentes roles y desafíos que el sistema de salud, la sociedad y la profesión demanda, así como de implementar diseños sólidos para la sostenibilidad de procesos de empoderamiento $(4,5)$.

Al respecto, aportes de diferentes autores mencionan que el empoderamiento en la educación, se da a raíz de los trabajos realizados por Paulo Freire y su pedagogía liberadora, que sostiene un enfoque participativo de la educación, en el que, las personas son los actores fundamentales del proceso de transformación $(6,7)$.

Así mismo, estudios de investigación demuestran que, el empoderamiento en enfermería tiene una relación significativa 
con la satisfacción laboral, elemento clave del clima organizacional y que los programas educativos de empoderamiento facilitan el trabajo en equipo, el aprendizaje, la comunicación adecuada, procesos de mejora continua, mayor cultura de seguridad del paciente y calidad de atención $(8,9)$.

En la misma línea, otros estudios evidencian que el empoderamiento es un método eficaz que permite a las personas enfrentar las presiones emocionales del entorno y es un pilar fundamental del desarrollo organizacional, mejora el poder y toma de decisiones en enfermería, a su vez correlaciona con la valentía moral, que es considerada como un valor básico de la profesión, sin embargo, implementar procesos de empoderamiento en las organizaciones es un gran desafío, debido a que todavía predomina un modelo de gestión burocrático en los diferentes escenarios que debilita las capacidades, limita el trabajo, el compromiso organizacional y profesional (1011).

Por tanto, un factor esencial en la formación de profesionales de enfermería, es el impulso de nuevos paradigmas para el desarrollo de prácticas de empoderamiento y un adecuado clima organizacional, que permita a los estudiantes desarrollar sus capacidades, sentido crítico, protagonismo, seguridad y visión de futuro, empoderándolos para promover cambios efectivos en las diferentes situaciones en que se desenvuelven y posicionar la identidad o imagen de la profesión en la sociedad, lo que se debe reflejar durante toda su formación y transcender al ejercicio profesional (12-14), a través de un perfil de egreso que supere las expectativas del sector salud y la sociedad para lograr un trabajo atractivo, significativo y participación activa en la toma de decisiones en los diferentes escenarios (15).

Por ello el objetivo del estudio es determinar la relación que existe entre empoderamiento y clima organizacional en estudiantes universitarios de enfermería.

\section{MATERIALES Y MÉTODOS}

Es una investigación con enfoque cuantitativo de tipo correlacional, transversal, diseño no experimental, desarrollada en la ciudad de Huancayo, durante los meses de marzo a diciembre de 2018. La población estuvo conformada por estudiantes del IV al IX semestre de la Facultad de Enfermería de la Universidad Nacional del Centro del Perú, constituida por 189 estudiantes. La muestra fue 166 estudiantes, la técnica de muestreo empleada fue probabilística aleatoria simple.

Para la recolección de datos de ambas variables, se usó como técnica la encuesta a través del cuestionario, previo consentimiento informado. Se aplicó los instrumentos durante los meses de junio y julio del 2018 en el horario de tutoría, se aseguró la confidencialidad y anonimato de los estudiantes. En cuanto al procesamiento y análisis de los datos se utilizó el paquete estadístico IBM SPSS Statistics 24.0, a través del cual se aplicaron estadísticos descriptivos, para obtener e interpretar las frecuencias absolutas y porcentuales de las 
variables de estudio y sus dimensiones, así como de la estadística inferencial para comprobar la validez de la hipótesis de investigación mediante la interpretación del p-valor del coeficiente de correlación de Pearson ( $r$ ) para un nivel de significancia $\alpha=0,05$. El estudio fue aprobado por el Centro de Investigación de la Universidad Nacional del Centro de Perú y se ejecutó con base a los principios éticos de beneficencia, no maleficiencia, justicia y autonomía.

\section{RESULTADOS Y DISCUSIÓN}

Los participantes, fueron 166 estudiantes de la Facultad de Enfermería, el $92,77 \%$ de sexo femenino, comprendidas entre las edades de 19 a 25 años, con una edad promedio de 23,90 años. El $84,34 \%$ de estudiantes, perciben que el clima organizacional es medianamente favorable, lo cual puede ser considerado como una brecha para el desarrollo del estudiante y de la organización (Tabla 1).

Tabla 1. Percepción de los estudiantes de enfermería sobre el clima organizacional.

\begin{tabular}{lccc}
\hline CLIMA ORGANIZACIONAL & FRECUENCIA & PORCENTAJE & $\begin{array}{c}\text { PORCENTAJE } \\
\text { ACUMULADO }\end{array}$ \\
\hline No favorable & 12 & 7,23 & 7,23 \\
Medianamente favorable & 140 & 84,34 & 91,57 \\
Favorable & 14 & 8,43 & 100,00 \\
\hline TOTAL & $\mathbf{1 6 6}$ & $\mathbf{1 0 0 , 0}$ & \\
\hline
\end{tabular}

Lasdimensionesconsideradasenlavariable clima organizacional, fueron: administración y gestión institucional, liderazgo y toma de decisiones, trabajo académico, identidad y pertenencia, comunicación institucional, ambiente estudiantil y condiciones de desarrollo académico.

Respecto a las dimensiones administración y gestión institucional, así como liderazgo y toma de decisiones el $30,13 \%$ y el $27,11 \%$ de los estudiantes, perciben como no favorable para el desarrollo personal y organizacional, mientras que el $57,22 \%$ y el $64,45 \%$ tienen una precepción medianamente favorable sobre las mismas dimensiones. Sobre el trabajo académico y la identificación y pertenencia con la institución el $66,27 \%$ y $63,25 \%$ de estudiantes consideran que es favorable, estos resultados permiten inferir que la oferta curricular, la formación integral, la vinculación y servicios de apoyo académico, cumplen con las expectativas de la mayoría de ellos (Tabla 2). 
Tabla 2. Percepción del estudiante sobre dimensiones del clima organizacional.

\begin{tabular}{lcccccccc}
\hline $\begin{array}{c}\text { Clima } \\
\text { Organizacional }\end{array}$ & \multicolumn{2}{c}{$\begin{array}{c}\text { Administración y } \\
\text { Gestión institucional }\end{array}$} & \multicolumn{2}{c}{$\begin{array}{c}\text { Liderazgo y toma de } \\
\text { decisiones }\end{array}$} & \multicolumn{2}{c}{$\begin{array}{c}\text { Trabajo } \\
\text { Académico }\end{array}$} & \multicolumn{2}{c}{$\begin{array}{c}\text { Identidad y } \\
\text { pertinencia }\end{array}$} \\
& $\mathbf{F}$ & $\mathbf{\%}$ & $\mathbf{F}$ & $\mathbf{\%}$ & $\mathbf{F}$ & $\mathbf{\%}$ & $\mathbf{F}$ & $\mathbf{\%}$ \\
\hline No favorable & 50 & 30,13 & 45 & 27,11 & 4 & 2,41 & 4 & 2,42 \\
Mediamente favorable & 95 & 57,22 & 107 & 64,45 & 52 & 31,32 & 57 & 34,33 \\
Favorable & 21 & 12,65 & 14 & 8,44 & 110 & 66,27 & 105 & 63,25 \\
\hline Total & $\mathbf{1 6 6}$ & $\mathbf{1 0 0 , 0 0}$ & $\mathbf{1 6 6}$ & $\mathbf{1 0 0 , 0 0}$ & $\mathbf{1 6 6}$ & $\mathbf{1 0 0 , 0 0}$ & $\mathbf{1 6 6}$ & $\mathbf{1 0 0 , 0 0}$ \\
\hline
\end{tabular}

Referente a las dimensiones, comunicación institucional, ambiente estudiantil y condiciones de desarrollo académico, el $77,71 \% ; 73,49 \%$ y $77,11 \%$ de los estudiantes perciben como medianamente favorable, lo que implica que se debe dar mayor énfasis a las relaciones interpersonales entre estudiantes y docentes, así como a las actividades académicas motivadoras e incluyentes, para garantizar una educación de calidad. (Tabla 3).

Tabla 3. Percepción del estudiante sobre dimensiones del clima organizacional.

\begin{tabular}{lcccccccc}
\hline $\begin{array}{c}\text { Clima } \\
\text { Organizacional }\end{array}$ & \multicolumn{2}{c}{$\begin{array}{c}\text { Administración y } \\
\text { Gestión institucional }\end{array}$} & \multicolumn{2}{c}{$\begin{array}{c}\text { Liderazgo y toma de } \\
\text { decisiones }\end{array}$} & \multicolumn{2}{c}{$\begin{array}{c}\text { Trabajo } \\
\text { Académico }\end{array}$} & \multicolumn{2}{c}{$\begin{array}{c}\text { Identidad y } \\
\text { pertinencia }\end{array}$} \\
& $\mathbf{F}$ & $\mathbf{\%}$ & $\mathbf{F}$ & $\mathbf{\%}$ & $\mathbf{F}$ & $\mathbf{\%}$ & $\mathbf{F}$ & $\mathbf{\%}$ \\
\hline No favorable & 17 & 10,24 & 24 & 14,46 & 30 & 18,07 & 4 & 2,42 \\
Mediamente favorable & 129 & 77,71 & 122 & 73,49 & 128 & 77,11 & 57 & 34,33 \\
Favorable & 20 & 12,05 & 20 & 12,05 & 8 & 4,82 & 105 & 63,25 \\
\hline Total & $\mathbf{1 6 6}$ & $\mathbf{1 0 0 , 0 0}$ & $\mathbf{1 6 6}$ & $\mathbf{1 0 0 , 0 0}$ & $\mathbf{1 6 6}$ & $\mathbf{1 0 0 , 0 0}$ & $\mathbf{1 6 6}$ & $\mathbf{1 0 0 , 0 0}$ \\
\hline
\end{tabular}

El $58,43 \%$ de estudiantes perciben que tienen un nivel medio de empoderamiento, y el $39,16 \%$ un nivel alto, lo que significa que asumen responsabilidades, toman decisiones, lideran en diferentes espacios, asumen roles importantes dentro de la facultad y muestran capacidades para participar en forma activa, en los procesos de formación profesional. (Tabla 4). 
Tabla 4. Empoderamiento en estudiantes de enfermería.

\begin{tabular}{|c|c|c|c|}
\hline Empoderamiento en estudiantes & Frecuencia & Porcentaje \% & Porcentaje acumulado \\
\hline Bajo & 4 & 2,41 & 2,41 \\
\hline Medio & 97 & 58,43 & 60,84 \\
\hline Alto & 65 & 39,16 & 100,00 \\
\hline TOTAL & 166 & 100,00 & \\
\hline
\end{tabular}

El $80,73 \%$ de estudiantes consideran tener un empoderamiento individual alto, habilidad que tienen para crear, liderar, mantener, modificar e influenciar de manera voluntaria a las personas, con la finalidad de alcanzar metas comunes en beneficio de la organización, a su vez el 78,31\% de estudiantes presentan empoderamiento organizacional alto, al identificar trabajo colaborativo con los directivos y docentes que ayuden al logro de objetivos y metas académico-institucionales (Tabla 5).

Tabla 5. Empoderamiento individual y organizacional en estudiantes de enfermería.

\begin{tabular}{lcccc}
\hline $\begin{array}{c}\text { Empoderamiento } \\
\text { en estudiantes }\end{array}$ & Empoderamiento individual & \multicolumn{2}{c}{$\begin{array}{c}\text { Empoderamiento organizacional } \\
\text { Porcentaje } \\
\mathbf{\%}\end{array}$} & $\begin{array}{c}\text { Frecuencia } \\
\text { Porcentaje } \\
\mathbf{\%}\end{array}$ \\
\hline Bajo & $\mathbf{2}$ & 1,20 & 13 & 7,83 \\
Medio & 30 & 18,07 & 23 & 13,86 \\
Alto & 134 & 80,73 & 130 & $\mathbf{7 8 , 3 1}$ \\
\hline TOTAL & $\mathbf{1 6 6}$ & $\mathbf{1 0 0 , 0 0}$ & $\mathbf{1 6 6}$ & $\mathbf{1 0 0 , 0 0}$ \\
\hline
\end{tabular}

Los resultados finales, indican que existe una relación directa entre el clima organizacional y empoderamiento de los estudiantes de la Facultad de Enfermería. Se aplicó la prueba de coeficiente $r$ de Pearson, se obtuvo $(0,335)$, significativa $(p=0,000<$ 0,05). (Tabla 6). 
Tabla 6. Clima organizacional y empoderamiento en estudiantes de enfermería.

\begin{tabular}{lcccc}
\hline \multirow{2}{*}{ CLIMA ORGANIZACIONAL } & EAJO & EMPODERAMIENTO & ALTO & TOTAL \\
\hline No Favorable & 2 & 10 & 0 & 12 \\
& $1,20 \%$ & $6,02 \%$ & $0,0 \%$ & $7,23 \%$ \\
Medianamente Favorable & 2 & 84 & 54 & 140 \\
& $1,20 \%$ & $50,60 \%$ & $32,53 \%$ & $84,33 \%$ \\
Favorable & 0 & 3 & 11 & 14 \\
& $0,0 \%$ & $1,81 \%$ & $6,63 \%$ & $8,44 \%$ \\
TOTAL & 4 & 97 & 65 & 166 \\
\hline & $\mathbf{2 , 4 1 \%}$ & $\mathbf{5 8 , 4 3 \%}$ & $\mathbf{3 9 , 1 6 \%}$ & $\mathbf{1 0 0 , 0 0 \%}$ \\
\hline
\end{tabular}

\section{Discusión}

El clima organizacional, es un ambiente percibido por sus miembros como un medio que facilita o dificulta el trabajo como refiere Goncalves (16). Para Chiavenato (17) es la cualidad o propiedad del ambiente organizacional que perciben o experimentan los docentes y estudiantes y que influye en su comportamiento. Por lo tanto, al realizar la valoración del clima organizacional, se determina el tipo de dificultades que existen a nivel del capital humano e institucional lo que facilita o dificulta los procesos de empoderamiento individual del estudiante. Un clima organizacional óptimo logra la satisfacción de sus docentes y estudiantes, y esto repercute sobre la calidad educativa (18).

Al respecto, el $84.34 \%$ de los estudiantes perciben un clima organizacional medianamente favorable, al realizar un análisis crítico y reflexivo de los resultados, estos se consideran como una barrera para el desarrollo de la organización, ya que los estudiantes son el principal factor estratégico en las entidades formadoras. Entre las dimensiones más valoradas están el trabajo académico, la identificación y pertenencia con $66,27 \%$ y el $63,25 \%$ aspectos que fortalece y los empodera en su desarrollo académico. Al ser identificado y considerado el clima organizacional, como un factor favorable según percepción del estudiante, permite inferir que facilita su rol y su liderazgo político como futuro profesional.

Sobre empoderamiento, Cordón manifiesta que es un término muy usado y poco comprendido, por lo mismo, cuesta mucho implementarlo, pero en realidad consiste en delegar autoridad y responsabilidad, al otorgar a los integrantes de una organización el sentimiento de que son dueños de su propio trabajoyresultados.ConcuerdaconMorales(20) al afirmar que el empoderamiento contempla dos dimensiones, la de posesión del poder, y la del proceso o aprendizaje, para acceder a este. Por otro lado, Bacqué (21) manifiesta, que el empoderamiento se puede ver a través de tres modelos adoptados por los distintos 
políticos y económicos actuales: el radical que tiene como objetivo la transformación social y se basa en el cuestionamiento del sistema capitalista; el socio liberal que articula la defensa de las libertades individuales con una atención a la cohesión social y a la vida de las comunidades y el neoliberal donde la economía del mercado se presenta como la solución a todos los problemas, donde se incluye la desaparición del Estado, así mismo, no cuestiona la desigualdad social o la pobreza, plantea gestionarla. En esta línea de ideas el empoderamiento de estudiantes durante su formación profesional debe ser con responsabilidad social, ciudadanía y ética, que propicie grados de libertad para que sean capaces de tomar decisiones orientadas al desarrollo de la profesión y la sociedad.

Los resultados muestran que el $58,43 \%$ y el $39.16 \%$ de estudiantes perciben que tienen un nivel medio y alto de empoderamiento. Al considerar la importancia de este, coincidimos con el punto de vista que expone Morales y otros (22) cuando afirman que los nuevos paradigmas en la formación universitaria han puesto un nuevo reto al docente al reconocer un empoderamiento del estudiante quien posee mayor información y es capaz de seleccionar a las universidades que mejor se ajustan a sus necesidades y expectativas, en este sentido Hernández (23) complementa al referir que lo principal en la formación es el desarrollo de pensamiento crítico y reflexivo con base en el conocimiento científico, tecnológico y humano, por lo cual, se deben propiciar experiencias de aprendizaje que potencie las habilidades y destrezas del estudiante para disfrutar de lo aprendido, todo esto solo puede ofrecer una organización empoderada y con clima favorable, que tiene como misión la formación integral del estudiante, al generar oportunidades para perfeccionar sus capacidades e intereses académicos y valorar sus logros individuales y organizacionales.

Respecto a la correlación del clima organizacional y empoderamiento de estudiantes de la Facultad de Enfermería, los resultados determinan que existe una relación directa. Es importante generar políticas y estrategias orientadas a fortalecer el clima organizacional para lograr el empoderamiento personal y colectivo de los estudiantes en diferentes escenarios. Al comparar con resultados de estudios de investigación, Choque (24) afirma que el clima organizacional influye en el empoderamiento de las enfermeras jefes, por otro lado, Zegarra (25) en un estudio en enfermeras encontró que existe correlación baja $(r=0,318)$ entre el clima organizacional y el empoderamiento psicológico, porque el clima organizacional fue percibido como no saludable y el empoderamiento psicológico fue de moderado a alto. Concordante con el autor anterior Quesada (26) refiere que el empoderamiento"posee un sentido psicológico del control o de la influencia personal y social" y parte de la premisa que "aprender a aprender" es la base del aprendizaje, y que es un proceso de concienciación que potencia las capacidades, todo esto en un escenario académico colaborativo con impacto positivo según Guerra y otros (27) En esa línea 
Marrero (28) propone una estrategia clave de empoderamiento para los estudiantes la elaboración de un "plan de vida personalizado y un plan de comunicación interna", esto llevará a la mejora de la productividad intelectual y el compromiso con su formación, al respecto Molina(29)señala que los estudiantes aprenden y se motivan al desarrollar su razonamiento en la reconstrucción y problematización de situaciones de su interés, es en este momento que el docente juega un papel de relevancia al ser facilitador del aprendizaje al fortalecer "competencias sólidas y eficaces para la transformación intelectual, social y humana" como expresa Damasceno y Ribeiro (30). Por lo expuesto, se puede deducir que como docentes universitarios nos encontramos frente a un gran reto para empoderar al estudiante, en un contexto en el que se ha modificado la forma de enseñar, aprender y evaluar con la utilización de nueva tecnología, dar asistencia y acompañamiento que genere confianza y motivación al estudiante más allá del cumplir con el horario de clase, aspecto claves para un nuevo rol.

Román et al., (31) señalan que el apoyo organizacional ejerce una influencia indirecta positiva sobre la participación en actividades de desarrollo de los miembros de la organización y sobre los comportamientos proactivos, así mismo, actúa como mediador de dicha relación, sugiere la promoción de climas organizacionales caracterizados por el apoyo y el empoderamiento. Esto refuerza, que un trabajo planificado toma en cuenta el clima organizacional efectivo y el empoderamiento del estudiante de enfermería, lo que potenciaría su desarrollo humano.

Una de las limitaciones es que la investigación se realizó en una universidad pública, por lo que los resultados son aplicables a esta población o poblaciones similares. Es importante replicar este estudio en otros espacios para generalizar los resultados obtenidos. Sin embargo, los hallazgos brindan evidencia científica para aplicar procesos de mejora continua en la formación de profesionales en enfermería.

\section{CONCLUSIONES}

Se concluye que el clima organizacional de los estudiantes de la Facultad de Enfermería fue medianamente favorable y el empoderamiento que ellos tienen es de tipo medio en su mayoría. También que existe una relación directa entre el clima organizacional y el empoderamiento de los estudiantes de la Facultad de Enfermería.

\section{REFERENCIAS BIBLIOGRÁFICAS}

1. MINSA. Documento Técnico Lineamientos de Política de Recursos Humanos en Salud 20212030. [Internet] 2021 [citado 16 abr 2021]. Disponible en: https://www.gob.pe/institucion/ minsa/normas-legales/235756-1357-2018minsa

2. Agrazal J, Ortega B. Satisfacción de estudiantes de enfermería con formación teórico-práctica. Universidad de Panamá, Sede Azuero. Enferm Univers [Internet] 2018 [citado 16 abr 2021]; 15(3):265-73. Disponible en: http://www.scielo.org.mx/pdf/eu/v15n3/23958421-eu-15-03-265.pdf 
3. Ojeda R, Podestá L, Ruiz R. Clima organizacional y cultura de seguridad de enfermería en un Instituto de Salud Materno Perinatal. Rev. cuba. enferm. [Internet] 2021 [citado 26 Jul 2021]; 37(2): [aprox. 0 p.]. Disponible en: http://www.revenfermeria.sld. cu/index.php/enf/article/view/3596

4. Mulens I, Consuegra $M$, Castellanos $G$, Ayala A. El modelo de empoderamiento en el proceso de enseñanza aprendizaje en la carrera licenciatura en enfermería. Facultad de Enfermería Lidia Doce, La Habana. Cuba. Facultad de Tecnología de la Salud. Revista Cubana de Tecnología de la Salud [Internet] 2016 [citado 17 abr 2021]. Disponible en: https:// www.medigraphic.com/pdfs/revcubtecsal/cts2016/cts163c.pdf

5. Diraditsile, K. Programas de empoderamiento juvenil en Botswana: ¿sobrestimados o poco investigados? Glob Soc Welf. [Internet] 2021 [citado 17 abr 2021]. Disponible en: https://doi. org/10.1007/s40609-020-00200-5

6. Hur M. Empoderamiento. En: Gellman MD (eds) Encyclopedia of Behavioral Medicine. Springer, Cham. [Internet] 2020 [citado 17 may 2021]. https://doi.org/10.1007/978-3-03039903-0_312

7. Cruz E. La educación transformadora en el pensamiento de Paulo Freire. Educere [Internet] 2020 [citado 17 Dic 2020]; 24(78):197206. Disponible en: https://www.redalyc.org/ jatsRepo/356/35663284002/html/index.html

8. Li H , Shi Y, Li Y, Xing Z, Wang S, Ying J et al. Relación entre el empoderamiento psicológico de la enfermera y la satisfacción laboral: una revisión sistemática y un metaanálisis. JAN Leading Global Nursing Research [Internet] 2018 [citado 17 jul 2021]; 74(6):1264-1277. Disponible en: https://doi.org/10.1111/ jan.13549

9. Amiri M, Khademian Z, Nikandish R. El efecto del programa educativo de empoderamiento de enfermeras en la cultura de seguridad del paciente: un ensayo controlado aleatorio. BMC Med Educ [Internet] 2018 [citado 17 jul 2021].
Disponible en: https://doi.org/10.1186/s12909018-1255-6

10. Cougot B, Gauvin J, Gillet N, et al. Impacto a los dos años de una intervención sobre el empoderamiento entre los equipos de atención médica: protocolo de estudio de un ensayo controlado aleatorio en un gran hospital universitario francés. BMC Health Serv Res [Internet] 2019 [citado 17 jul 2021]. Disponible en: https://doi.org/10.1186/s12913-019-4724-7

11. Khoshmehr Z, Barkhordari-Sharifabad $M$, Nasiriani $K$, et al. Coraje moral $y$ empoderamiento psicológico entre enfermeras. BMC Nurs [Internet] 2020 [citado 21 jul 2021]; 19, 43. Disponible en: https://doi. org/10.1186/s12912-020-00435-9

12. Villagra $N$, Ruoti $M$. Empoderamiento de enfermería en las unidades de cuidados intensivos. Hospital Central del Instituto de Previsión Central. Rev. cient. estud. investig. [Internet] 2018 [citado 21 jul 2021]; 60:1402. Disponible en: http://revista.unibe.edu.py/ index.php/rcei/article/view/20

13. Blancas E. Educación y desarrollo social. [Internet]. Horizonte De La Ciencia [Internet]2018 [citado 21 jul 2021]; 8(14): 113121. Disponible en: https://revistas.uncp.edu. pe/index.php/horizontedelaciencia/article/ view/298

14. Rojas $L$. El fenómeno del poder en enfermería en la academia y el área asistencial desde la perspectiva del docente. Rev. Horizonte Sanitario [Internet] 2019 [citado 21 jul 2021]; 18(1). Disponible en: https://doi.org/10.19136/ hs.a18n1.2067

15. Iglesias A, Esperón J, SolísF.Unacercamiento al clima organizacional. Rev Cubana Enferm [Internet] 2018 [citado 9 Jun 2021]; 34(1): Disponible en: http://www.revenfermeria.sld. cu/index.php/enf/article/view/1257

16. Gonçalves P. Dimensiones del clima organizacional. Sociedad Latinoamericana para la Calidad (SLC). [Internet]. 2018. [citado 29 oct 2018]; Disponible en: http://www. geocities.ws/janethqr/liderazgo/130.html 
17. Chiavenato I. Administración de recursos humanos. El capital humano de las organizaciones. [Internet]. 2007. [citado 30 nov 2018]; Disponible en: https:// cucjonline.com/biblioteca/files/original/ aec4d0f8da9f45c14d9687966f292cd2.pdf

18. Bada F, Salas R, Castillo E, Arroyo E, Carbonell C. Estrés laboral y clima organizacional en docentes peruanos. Medisur [Internet] 2020 dic. [citado 18 may 2021]; 18 (6): 1138-1144. Disponible en: http://scielo.sld. cu/scielo.php?script=sci_arttext\&pid=S1727897X2020000601138\&lng=es

19. Cordón V. (2015). ¿Como empoderar a mis colaboradores? Grupo Perspectiva, S.A. [Internet] 2017. [citado 18 may 2021]. Disponible en: http://recursosbiblio.url.edu.gt/ tesisjcem/2017/05/43/Valdez-Monica.pdf

20. Morales E. Empoderamiento y transformación de las relaciones de poder. Un análisis crítico de los procesos institucionales de participación ciudadana. [Internet]. Universitat Autónoma de Barcelona. 2016. [citado 14 may 2021]. Disponible en: https:// www.tdx.cat/bitstream/handle/10803/400078/ emmldel.pdf?sequence $=1$

21. Bacqué M, Biewener C. El empoderamiento. Una acción progresiva que ha revolucionado la política y la sociedad. [Internet] Gedisa. Barcelona. 2016. 164 pp. ISBN: 978-84-9784846-6. [citado 14 may 2021]; Disponible en: https://dialnet.unirioja.es/servlet/ articulo?codigo $=6664079$

22. Morales $M$, Balcázar $C$, Priego $H$, Flores J. El empoderamiento del alumno: una tendencia favorable en la educación superior. Revista Iberoamericana para la Investigación y el Desarrollo Educativo [Internet] 2021 [citado 29 jun 2021]. Disponible en: https://www.ride.org.mx/index.php/RIDE/ article/view/847/2977\#content/citation_ reference_14

23. Hernández $R$, Infante $M$. La clase en la educación superior, forma organizativa esencial en el proceso de enseñanza- aprendizaje. Educación y Educadores. Pedagogía Universitaria [Internet] 2017 [citado 20 jul 2021]; 20(1): 27-40. Disponible en: http:// www.scielo.org.co/pdf/eded/v20n1/01231294-eded-20-01-00027.pdf

24. Choque T. Influencia del Clima Organizacional en el empoderamiento en jefaturas de enfermería de los centros de salud de la Red Arequipa-Caylloma 2018. [Internet]. [citado 30 dic 2018]; Disponible en: URI: http:// repositorio.unsa.edu.pe/handle/UNSA/8630

25. Zegarra R. Relación entre clima organizacional y empoderamiento psicológico en enfermeras que brindan cuidado a personas afectadas por tuberculosis en la Red de salud Rímac-SMP-LO. [Internet]. 2015. [citado 14 may 2021]. Disponible en: https://repositorio.ucv. edu.pe/handle/20.500.12692/6782

26. Quesada A. Empoderamiento del alumnado en su aprendizaje. [Internet]. Red Social Educativa. 2017. [citado 14 jun 2021]. Disponible en: https://redsocial.rededuca.net/ node/6065

27. Guerra M, Rodríguez J, RodríguezJ. Aprendizaje colaborativo: experiencia innovadora en el alumnado universitario. REXE, Revista de Estudios y Experiencias en Educación [Internet]2019. [citado 14 jul 2021]; 18(36): 269-281. Disponible en: http:// www.rexe.cl/ojournal/index.php/rexe/article/ view/661

28. Marrero $M$. El empoderamiento en el entorno empresarial. [Internet]. 2018. [citado 30 diciembre 2020]. Disponible en: https://momomarrero.com/2018/03/19/elempoderamiento-en-el-entorno-empresarial/

29. Molina M. Prácticas de enseñanza que entraman escritura con contenidos: perspectivas de alumnos universitarios de dos disciplinas. Propuesta Educativa [Internet] 2018 [citado 10 jul 2021]; 1(49): 6172. Disponible en: https://www.redalyc.org/ jatsRepo/4030/403060198007/html/index. html 
30. Damasceno E, Ribeiro de Lira M. Formación de profesores, plan de estudios y práctica pedagógica: enseñanza interdisciplinaria. Educere [Internet]2018[citado 10 jul 2021]: 22(72), 283-293. Disponible en: https://www. redalyc.org/jatsRepo/356/35656041002/html/ index.html

31. Román-Calderón J, Krikorian A, Franco C, Betancur A. Apoyo organizacional y empoderamiento como antecedentes de comportamientos empoderados y participación de los empleados Journals \& Books. Science Direct. Estudios Gerenciales. Universidad ICESI [Internet] 2016 [citado 14 de mayo 2021]; 32(139): 154-161. Disponible en: http://dx.doi.org/10.1016/j.estger.2016.03.002

Conflicto de intereses: Ninguno declarado por los autores. Financiación: Ninguna declarada por los autores.

Agradecimiento: Ninguno manifestado por los autores

\section{ACERCA DE LOS AUTORES}

Rossana Mirtha Scarsi Maratuech. Doctora en Ciencias de la Salud y Salud Pública. Magister en Desarrollo Rural. Licenciada en enfermería. Docente de pregrado y posgrado. Universidad Nacional del Centro del Perú, Perú.

Guillermina Delia Pérez Gutarra. Magister en Desarrollo Rural. Licenciada en enfermería. Docente de pregrado y postgrado. Universidad Nacional del Centro del Perú, Perú.

Carolina Mercedes Cristobal Tembladera. Maestría en Docencia e Investigación en Educación Superior. Doctorado en Ciencias de la Educación Universidad Enrique Guzmán y Valle. Docente Universitario, Universidad Continental, Universidad del Centro del Perú, Perú. 